\title{
Fixation of the Cemented Stem: Clinical Relevance of the Porosity and Thickness of the Cement Mantle
}

\author{
Philippe Hernigou ${ }^{*}, 1$, Gildasio Daltro ${ }^{2}$, Charles Henri Flouzat Lachaniette ${ }^{1}$, Xavier Roussignol $^{1}$, \\ Martin Mukisi Mukasa ${ }^{3}$ and Alexandre Poignard ${ }^{1}$ \\ ${ }^{1}$ Hôpital Henri Mondor - 94010 CRETEIL, France \\ ${ }^{2}$ Hospitals Edgar Santos, University Federal of Bahia, Salvadore, Brazil \\ ${ }^{3}$ Hospital de Point à Pitre- Guadeloupe, France
}

\begin{abstract}
The aim of this review paper is to define the fixation of the cemented stem. Polymethyl methacrylate, otherwise known as "bone cement", has been used in the fixation of hip implants since the early 1960s. Sir John Charnley, the pioneer of modern hip replacement, incorporated the use of cement in the development of low frictional torque hip arthroplasty. In this paper, the concepts of femoral stem design and fixation, clinical results, and advances in understanding of the optimal use of cement are reviewed. The purpose of this paper is to help understanding and discussions on the thickness and the porosity of the cement mantle in total hip arthroplasty. Cement does not act as an adhesive, as sometimes thought, but relies on an interlocking fit to provide mechanical stability at the cement-bone interface, while at the prosthesis-cement interface it achieves stability by optimizing the fit of the implant in the cement mantle, such as in a tapered femoral stem.
\end{abstract}

\section{INTRODUCTION}

Despite being in clinical use for more than 35 years, the success of cemented femoral stems is still not completely understood. Many reports have demonstrated good long term results with cemented stems. Therefore it seems justified to still use cemented stems in all age groups of patients.

Nevertheless the importance of thickness, voids, porosity, homogeneity and extent of the femoral cement are still controversial. Several variables influence the shape of the cement mantle, the reproducibility and the quality of the cement mantle. Furthermore the adequacy of the cement mantle and the cement-implant and cement-bone interfaces are probably factors affecting the longevity of cemented femoral implants. Many cemented stems show micromotions exceeding $0.1 \mathrm{~mm}$ during the first two years when the measurement is done with radiostereometric analysis. In most of the studies these motions occur inside the cement mantle. However the cement mantle can also be displaced in relation to the bone. The micromotions of the stem may reflect a deformation of the cement mantle or a complete debonding of the stem.

Failure of the cement mantle, when it occurs, can lead to mantle fracture; stem debonding, osteolysis and loosening. The failure $[1,2]$ can be initiated at one or more of the following regions: the cement-bone interface, the cement and the cement-prosthesis interface. Factors that influence can be broken into implant factors (geometry, surface finish,

\footnotetext{
*Address correspondence to this author at the Department of Orthopaedics Surgery, University Paris XII, Hospital Henri Mondor, 94010 Creteil, France; Tel: 33149812601; Fax: 33149812608;

E-mail: philippe.hernigou@wanadoo.fr
}

neck length), cement factors (porosity, thickness), technical factors (bone preparation, instrumentation, stem orientation) and patient factors (bone quality...). The purpose of this paper is to help understanding and further discussions on the thickness and the porosity of the cement mantle in total hip arthroplasty.

\section{THE THICKNESS OF THE CEMENT MANTLE?}

\subsection{Cement Mantle of $3 \mathrm{~mm}$ : Is it the Gold Standard?}

Traditionally, surgeons have adopted to obtain a mantle of at least $3 \mathrm{~mm}$ around the implant, as thin and deficient mantles have been associated with adverse results. Recently, this viewpoint has been challenged by Langlais et al. [3], who have fashioned the phrase "the French paradox" to describe their finding that certain French-designed straight stems [4], which maximally fill the medullary canal with the intention of leaving thin or even incomplete mantles in places, have good medium- and long-term results. Under these conditions, at certain points of the femoral stem the cement thickness is probably less than $3 \mathrm{~mm}$. They have reported good results with such a technique. However several authors, based on clinical, radiological, and histological analyses, have suggested that a thin cement mantle is subjected to increased strain may fragment. Since then, it has become perceived wisdom among surgeons who use acrylic cement that the thickness of the cement mantle important [5]. On first consideration, this conclusion seems justified. A number of experimental studies appear to demonstrate that the femoral cement mantle must be at least $3 \mathrm{~mm}$ thick and that the distal end of the femoral part should not occupy more than $80 \%$ of the width of the femoral canal, to reduce risks of cement fatigue fracture. This led to the assumption 
that measures that increase the thickness and the reproductibility of the cement mantle would increase the durability of the arthroplasty in clinical practice.

\subsection{Evaluation of the Cement Mantle}

If this was true, there should be no difficulty in showing that implants inserted with a large cement mantle survive better than those implanted with a thin cement mantle. Such a comparative study is still to be published. Probably because a thin cement mantle less than one millimeter is very frequent and because it is difficult to assess. Clinically the cement mantle is assessed by inspecting plain radiographs; and to standardize analysis, cement grading systems have been developed [6,7]. However the application of these systems is challenging. Analysis of plain films is difficult particularly with poor-definition of the cement-bone interface, and difficulty to identify voids. It seems that this degradation of information is very important. Probably, as reported by Kawate [8], plain radiographs miss areas of cement thinner than $1 \mathrm{~mm}$ particularly in the sagittal plane. The cement mantle is very thin in the anteromedial part of the proximal femur due to a mismatch between a straight femoral stem, the anteversion of the neck and the anterior bow of the proximal part of the femur. Autopsy studies [9] have demonstrated that routine clinical anteroposterior radiographs are deficient in accuracy in terms of identifying all the areas of the cement [10-12]. Lateral radiographs are better because they show more frequently impingement of the stem on the femoral cortex. CT scan frequently demonstrates a very thin mantle of cement. So the issue for debate is whether in clinical practice the thickness of the cement mantle affects the outcome of the stem. The report by Mulroy and Harris $[6,7]$ showed a significant correlation between a thin cement mantle as seen on radiographs and an increased risk of loosening. However in the series of Mulroy and Harris the definition of a thin cement mantle was not exactly a thin cement mantle between an adjusted stem and the bone (because for example the stem impinged on the bone); it was rather an absence of cement around a stem at a distance from the bone. The difference is important because in the first case it is a thin cement mantle with a very welladjusted stem in the femoral canal and the primary stability of the implant is good; in the second case it is a poor technically cemented thin stem in a large femoral canal and the primary stability of the implant is poor.

\subsection{Clinical Relevance of the Thickness of the Cement}

So Mulroy and Harris demonstrated that the absence of primary stability of the cemented implant affects the outcome of the stem but there was not in their report any clinical evidence that a thick cement mantle is better than a thin cement mantle when the primary stability of the stem is achieved. In considering this matter, not many studies have been reported. One study [13] has analyzed this problem on a series of 156 Ceraver Osteal hip replacements. The initial metaphyseal femoral index was measured on the X-Ray of the first year. It was on average $70.9 \%$ with a minimum of $50.6 \%$ and a maximum of $85.5 \%$. The average initial distal femoral index measured on the X-Ray of the first year was $80.4 \%$ (minimum $57.3 \%$, maximum $100 \%$ ). No loosening was observed in patients showing maximum femoral indices i.e. when the larger stem was implanted in the femoral canal. Conversely, in the event of poor filling of the femoral diaphysis by the implant, the number of radiolucent lines was higher with an increased risk of femoral loosening (2 loosenings). In addition, given the front curvature of the femur and the use of an identical right and left prosthesis, the use of the thickest possible prosthesis episodically resulted in contact between the distal end of the prosthetic stem and the front cortex of the femur. In these cases, the cement mantle may be considered to be non-existent at this point. This problem was encountered in approximately $7 \%$ of total hip replacements when a lateral X-Ray was performed on the femoral diaphysis [14]. This impingement between the end of the prosthesis and the anterior cortex of the femur was looked for on lateral X-Rays of prostheses reviewed after over 10 years; when present, this impingement did not result in any loosening. In view of this study, the thickness of the cement mantle [15] does not appear to have an influence on the risk of loosening after over 10 years. The possibility is that late loosening is related to an absence of primary stability of the implant rather than to the thickness of the mantle of cement. The cement mantle thickness does not affect the primary stability of the implant. If the thickness of the cement mantle had had an influence on the long-term stability of the implant, migration of the implant would have been expected after fatigue fracture of the cement (i.e. after about 6 to 8 years of follow-up). Had this occurred it would have been in contradiction with most of the studies $[16,17]$ on migration of implants which indicate that any femoral implant that migrates more than $2 \mathrm{~mm}$ in the first 2 years is likely to fail within 10 years, whether the implant is fixed with or without cement. This observation is important since it suggests that the factors leading to early and excessive migration (and consequent later loosening) do not differ between cemented and cementless implants and thus that these factors relate to the primary stability of the implant. This in turn means that late loosening is related to an absence of primary stability rather than to late fatigue behavior of the cement caused by insufficient thickness. This would explain the findings of Mulroy and Harris. Conversely, a prosthesis that is too small in a femoral shaft may result in several problems: more frequent lucent lines, cement mass introduced into the larger femoral shaft and, therefore, risk of greater retraction of cement during polymerization.

\subsection{Fixation of the Cement Mantle}

Now turning the discussion to another aspect of the cement mantle, one can ask what is the better fixation of the cement mantle on bone, particularly if the cement mantle is thin: is it cortical bone without penetration of the cement or trabecular bone with penetration of the cement [18]. The two parts of the cement mantle submitted to the most important forces are the upper medial part and the antero lateral distal part. For the distal part, the bone is cortical; for the medial part the question is: to remove or not the trabecular bone? Because micromotion of the cement mantle is a possibility due to the viscoelasticity of the cement, there is a risk of fracture of a thin cement mantle if the fixation is too good on the trabecular bone. If the bone is only cortical and smooth, a small thin cement mantle is able to creep under load, to transmit the load to the proximal femur and can subside at the bone-cement interface without loosening exactly like a 
polished stem can subside without loosening at the prosthesis-cement interface [19]. So the assumption that pressurization of the cement mantle in the trabecular bone [10] increases the durability of the arthroplasty may not be valid in clinical practice. And when the evidence is considered more carefully by surgeons using polished stems in a cement mantle to allow micromotion of the stem in the cement mantle, this assumption of pressurization of the cement mantle in the trabecular bone becomes more difficult to accept.

\subsection{Centralization of the Cement Mantle}

To eliminate the formation of a defective mantle or a too thin cement mantle at the distal part, some surgeons have proposed to use an automatic centralization of the stem within the canal. The benefits of an optimum cement mantle remain theoretical unless the stem can be positioned centrally within the cement mantle. Noble et al. [21] have proposed methods to control the position of the distal stem. Distal centralizers prevent bony impingement, but they can also cause accumulation of significant porosity and accumulation of blood and fat into the cement stem interface. Follow-up is necessary to know exactly the benefits of these solutions.

\section{VOIDS IN THE FEMORAL CEMENT MANTLE}

\subsection{Voids in Cement}

Cement contains voids as a direct consequence of the way it is prepared from monomer and pre-polymerized beads. Voids are usually regarded as deleterious to the mechanical strength of cement. At least on first consideration, this conclusion seems justified, when a piece suddenly breaks because it contains less matter per unit volume [22, 23]. Many experimental studies on this subject have investigated bone cement failures in the laboratory [24]. But to our knowledge, there are few reports on the influence of radiological voids in cement on the long term risk of loosening of total hip arthroplasties $[25,26]$. Different mechanical methods for preparing cement, with the aim of reducing the number of pores, have been proposed based on theoretical principles. These methods include using low-viscosity cement, mixing it under a vacuum, centrifugation during preparation [27-29], and even mixing it by ultrasound. The effect of increasing the temperature of the stem has been tested on the porosity at the cement-stem interface. It was proposed that with stems at room temperature, the polymerization starts at the warming bone-cement interface and proceeds centripetally toward the stem. The stem-cement interface cures last with accompanying pores and flaws. When heating the stem, the cement in contact with the stem polymerizes first and has significantly fewer pores and flaws. However it is difficult to find clinical studies on the risk of weakening and loosening associated with the presence, within cement mantles, of porous areas large enough to be seen on X-Rays, even if some reports $[14,26]$ have used classification systems looking at cement voids.

\subsection{Porosity of the Cement}

Several authors have reported that in vitro, reducing porosity improves the mechanical properties of the cement $[30,31]$. However, the experiments in vitro cannot simulate the clinical or the in vivo conditions of a cemented arthroplasty. Thus, these findings can only be interpreted as material properties related to bone-cement mixing techniques. These results are not demonstrated in clinical studies [32-34] and are furthermore in contradiction with some clinical data: for example, it is well known that voids are more frequent with high viscosity cement; however, in a study of the survival of 8579 arthroplasties, Havelin et al. [35] found that poorer results were observed when the femoral component was implanted with low viscosity cement. Mulroy et al. [6, 7] in a series of prostheses followed for a minimum of 14 years reported that there is no relationship between small voids in the bulk of cement and loosening but that loosenings were more frequent when large voids were observed; however he did not give any measure of the voids in the grades of the mantle of cement. Raut et al. [26] found that the presence of voids in the cement mantle had little influence on the results of revision using cemented prostheses, but no measure of voids was given. Ling and Lee [36] with a long term series of Exeter hip replacements found that porosity in acrylic cement was clinically without consequences. Hernigou and Le Mouël [37] reporting on a series of Ceravel Osteal stems implanted between 1983 and 1985 noted that the risk of revision was higher when the cement mantle of the femur had no porosity. Malcolm [38] in his study of retrieved Charnley replacements searched for cracks in the cement associated with pores, but gave up the search after examining 1000 pores without finding a crack associated with a pore.

\subsection{Clinical Relevance of Porosity in Cement}

There may be several explanations for the absence of any clinical relevance of porosity on the outcome of the stem:

The first explanation is that radiological voids in cement do not have a marked adverse effect on the mechanical properties of the cement mantle. This may seem surprising at first, but it is actually consistent with the mechanical data. In fact, from the mechanical viewpoint [39], a cylindrical tube which contains a hole is only marginally reduced in strength, as long as the diameter of the hole is less than $30 \%$ of the total diameter of the cylindered tube, as was the case for the radiological detected pores. This means that a cylindrical tube of $15 \mathrm{~mm}$ in diameter can accept a hole of $5 \mathrm{~mm}$ of diameter without reduction in strength. These apparent voids on X-Rays should, however, be distinguished from large irregular defects (greater than $10 \mathrm{~mm}$ ) situated at the periphery of the mantle of cement. In contrast to spherical or oval voids observed in our series, the dimensions of which rarely exceed 4 or $5 \mathrm{~mm}$, a large irregular defect entails a much greater reduction in the cement sleeve, and consequently, a much greater loss of its strength [25, 37-39]. The content of a void is usually gas. According to the experience of the authors, the cause of a large irregular cement defect is rather blood in the distal part of the cement mantle and rather the presence of cancellous bone in the proximal part. So, reduction of large defects in the cement is depending on preparation of the medullar canal by curetting cancellous bone and drying the medullar canal. In contrary, voids are rather a direct consequence of the way of preparation of the cement.

The second explanation is that voids in cement do have a small deleterious effect on the mechanical strength of the 
cement mantle, but it was impossible in most of the series to demonstrate a significant difference.

The third explanation could be that only very small voids or pores not detectable by eye have a deleterious effect on the mechanical strength of the cement mantle. This possibility exists since the number of micropores only detectable by electron microscopy [26] is greater than the number of voids detectable by X-Rays. The theoretical disadvantage of a micropore is not exactly the same as for a macropore or a void: with voids (macropores) the cement contains less matter per unit volume; with micropores, another debate [22] is whether in clinical practice, micropores in the cement are crack initiators, crack stoppers or have no influence on crack propagation. Mathematical models show that theoretical, breaking constraint increases with the sharpness of the tip of crack, the propagation of which leads to the breaking of the material. So a crack at an acute angle (as a micropore), even one of just a few microns, represents a far greater force constraint than a macro round hole (as a void), because of the likelihood of such a crack spreading. However when the crack tip progresses and runs into a round void, the crack tip naturally turns from an initially sharp crack into a blunt crack and it turns out from the mathematics that with crack blunting the breaking constraint decreases; so at the microscopic scale a pore can initiate a crack but also can stop a crack. Furthermore Roberts et al. [40] in a retrieval study of eleven cadaveric femora with cemented stems found that no cement fractures were in relation with areas of porosity within the cement mantle. So whether micropores in the cement are crack initiators or crack stoppers [41, 42] are a controversial matter; but there is no clinical evidence to date that a "pore-free" cement improves clinical results by comparison with cement that contains pores.

The problem is that "pore-free" cement might even be harmful. Herberts and Malchau [43] when reporting the results of the Swedish Hip registry have confirmed the results of Havelin [35], i.e., that poorer results are obtained when the femoral component was implanted with lowviscosity cement associated with vacuum mixing. When using a time dependent Poisson model, they found a higher risk for revision in the first four years with vacuum mixing, such a result probably also indicating an insufficient primary stability perhaps linked to technical mishandling or too early stem insertion with a low viscosity cement.

\section{THE DESIGN OF FEMORAL STEM}

This design [20, 45, 46] clearly influences the clinical result either by effects at the prosthesis-cement interface, or how the stem transfers load to cement and thereby to bone. The combination of shape and surface finish of the stem significantly influences long-term results. Based largely on the surface finish of the stem, and also on the way the stem interacts with the cement mantle, two main philosophies of fixation have evolved: the polished stem surface and the rough stem surface.

The first approach uses the highly polished surface to optimize fit of the stem within the cement mantle and ensure that wear due to micromotion at the prosthesis-cement interface is minimized. Of the polished stems, there are essentially three types, some of which have been successful over a number of decades. There are, first, those modeled on the original polished Charnley stem [44], which have a rounded stem proximally with relatively rounded edges in cross section; second, the collarless double-tapered stems, characterized by tapers from proximal to distal in the sagittal and frontal planes and a predominantly rectangular cross section proximally [14]; and third, and more recently, collarless triple-tapered stems, which have an additional taper from lateral to medial. The proposed reason for the excellent clinical results of the polished stems $[47,48]$ is that they subside within the cement mantle, usually by a few millimeters, and this mainly occurs over the first few years following insertion. Simply put, and remembering that stems are subject to considerable anteroposterior forces, this ensures the stem wedges itself tightly into the cement mantle, thereby ensuring optimal contact between the stem and cement. Under load, axial forces are converted by the taper in the cement to radially compressive forces at the cement-bone interface.

The other philosophy of stem design aims to achieve rigid interlock between the stem and cement and thereby nullify movement at this interface. This includes matte, gritblasted and beaded or porous surfaces and those with indentations [49]. A further development of this philosophy includes those stems manufactured with a precoat of cement applied to the stem with the aim of improving bonding between the stem and cement. It is proposed that the implantcement-bone construct acts as a "composite beam", and the intention is to prevent movement at each of the interfaces. For this composite beam to be effective there needs to be perfect bonding at both interfaces, with good support from the cement. If the construct is unable to withstand the stresses passing through it during loading, then either the cement mantle will fracture or undesirable movement will occur between implant and cement, leading to debonding at the interface. With this type of system, separation of the stem from cement, termed debonding, is thought to be an important event in the development of aseptic loosening. Analysis of cemented stems of the rough type retrieved postmortem has demonstrated separation of these stem types from cement and fractures within the cement. Thus, debonding of this design of stem is considered a predictor of failure, but this must be distinguished from the designed subsidence of polished tapered stems, which in fact is proposed to optimize fixation.

\section{DISCUSSION AND CONCLUSION}

Prior to modern cementing techniques [28], many good results with long term follow-up have been obtained in total hip arthroplasties. This does not mean that the cement porosity should not be avoided but only means that this porosity probably has a minor influence on the durability of the cement mantle and that probably "modern" cementing techniques will not improve significantly the outcome of femoral prostheses in the next decades. It will probably be difficult with "modern" cementing technique to obtain better results than those reported for the femur by some authors from very old operations (with more than twenty years follow-up) when using finger packing [44]. One of the efforts in the past decade has been to try to quantify what constitutes a so called good cementing technique versus a poor one. Many techniques have been developed and tried: insertion of an intramedullary plug, vacuum mixing, pulse lavage, pressuri- 
zation using a proximal seal, removal of any loose cancellous bone, drying with adrenaline-soaked sponges, and centralizers on the femoral stem... Probably, all these modern techniques have the advantage to explain how to achieve a good result with a routine basis in nearly every case. However one should remind first that some of these techniques incorporate ideas which are relatively untested and may end to an adverse effect; secondly, that an old cementing technique with finger packing has not prevented in the past many surgeons to get a femoral cement mantle good enough to allow excellent results in the long term for well-designed femoral stems.

\section{REFERENCES}

[1] Gruen TA, Mc Neice GM, Amstutz HC. "Modes of failures" of cemented stem-type femoral component. Clin Orthop 1979; 141: 17-27.

[2] Johnston RC, Fitzgerald R, Harris N, Poss R, Müller M, Sledge C. Clinical and radiologic evaluation of total hip replacement. J Bone Joint Surg 1990; 72-A: 161-8.

[3] Langlais F, Kerboull M, Sedel I, Ling RSM. The 'French Paradox'. J Bone Joint Surg Br 2003; 85-B: 17-20.

[4] Kerboull L, Hamadouche M, Courpied JP, Kerboull M. Long-term results of Charnley-Kerboul hip arthroplasty in patients younger than 50 years. Clin Orthop 2004; 418: 112-8.

[5] Skinner JA, Todo S, Taylor M, et al. Should the cement mantle around the femoral component be thick or thin? J Bone Joint Surg Br 2003; 85-B: 45-51.

[6] Mulroy RD Jr, Harris WH. The effect of improved cementing techniques on component loosening in total hip replacement. An 11-year radiographic review. J Bone Joint Surg 1990; 72-B: 75760.

[7] Mulroy W, Estok D, Harris W. Total hip arthroplasty with use of so called second generation cementing techniques. J. Bone Joint Surg 1995; 77-A: 1845-52.

[8] Kawate K, Mmaloney WJ, Bragdon BS. Importance of a thin cement mantle. Clin Orthop 1998; 355: 70-6.

[9] Hirakawa K, Jacobs JJ, Urban R, Saito T. Mechanisms of failure of total hip replacements: lessons learned from retrieval studies. Clin Orthop 2004; 420: 10-7.

[10] Valdivia GG, Dunbar MJ, Parker DA, et al. The John Charnley Award: three-dimensional analysis of the cement mantle in total hip arthroplasty. Clin Orthop 2001; 393: 38-51.

[11] Mellor SJ, Ripley LG, Ricketts DM. The femoral cement mantle in total hip replacement. Int Orthop 2004; 28: 40-3.

[12] Scheerlinck T, de Mey J, Deklerck R. In vitro analysis of the cement mantle of femoral hip implants: development and validation of a CT-scan based measurement tool. J Orthop Res 2005; 23: 698704

[13] Hernigou P. Femoral cement mantle thickness; its influence on the outcome of the femoral part. Acora Group Ed. Total hip arthroplasty 2000; pp. 183-6.

[14] Hernigou P. Twenty years experience with the cemented smooth titanium alloy stem Ceraver Osteal in primary cases. J Bone Joint Surg 1999; 81-B (Suppl II): 158.

[15] Ramaniraka NA, Rakotomanana LR, Leyvraz PF. The fixation of the cemented femoral component: effects of stem stiffness, cement thickness and roughness of the cement-bone interface. J Bone Joint Surg Br 2000; 82-B: 297-303.

[16] Freeman MAR, Plante Bordeneuve P. Early migration and late aseptic failure of proximal prosthesis. J Bone Joint Surg 1994; 76B: $432-8$.

[17] Kiss J, Murray DW, Turner-Smith AR, Bithell J, Bulstrode CJ. Migration of cemented femoral components after THR. J Bone Joint Surg 1996; 78B: 796-801.

[18] Ayers D, Mann K. The importance of proximal cement filling of the calcar region: a biomechanical justification. J Arthroplasty 2003; 18: 103-9.

[19] Huiskes R, Verdonschot N, Nivbrant B. Migration, stem shape, and surface finish in cemented total hip arthroplasty. Clin Orthop 1998; 355: $103-12$.
[20] Breusch SJ, Norman TL, Revie IC, et al. Cement penetration in the proximal femur does not depend on broach surface finish. Acta Orthop Scand 2001; 72: 29-35

[21] Noble PC, Collier MB, Maltry JA, Kamaric E, Tullos HS. Pressurization and centralization enhance the quality and reproducibility of cement mantles. Clin Orthop 1998; 355: 77-89.

[22] James SP, Jasb M, Davies J, Piehler H, Harris WH. A fractographic investigation of PMMA bone cement focusing on the relationship between porosity reduction and increased fatigue life. J Biomed Mater Res 1992; 26: 651-2.

[23] Janssen D, Stolk J, Verdonschot N. Why would cement porosity reduction be clinically irrelevant, while experimental data show the contrary. J Orthop Res 2005; 23: 691-7.

[24] Evans SL. Effects of porosity on the fatigue performance of polymethylmethacrylate bone cement: an analytical investigation. Proc Inst Mech Eng [H] 2006; 220: 1-10.

[25] Hernigou P, Pernod P. An ultramicroscopic study of cement porosity. Charnley total hip arthroplasty "33 years of vorldwilde experience". Group A.C.O.R.A. Ed., Lyon 1995.

[26] Raut VV, Siney PD, Wroblewski BM. Revision for aseptic stem loosening using the cemented Charnley prosthesis. J Bone Joint Surg 1995; 77-B: 23-7.

[27] Davies JP, Jasty M, O'Connor DO, Burke DW, Harrigan TP, Harris WH. The effect of centrifuging bone cement. J Bone Joint Surg Br 1989; 71: 39-42.

[28] Harris WH, Davies JP. Modern use of modern cement for total hip replacement. Orthop Clin North Am 1988; 19: 581-9.

[29] Saha S, Pal S. Mechanical properties of bone cement: a review. J Biomed MaterRes 1984; 18: 435-62.

[30] Chao ES, Chin HC, Stauffer R. Roentgenographic and mechanical performance of centrifuged cement in a simulated total hip arthroplasty model. Clin Orthop 1992; 285: 91-6.

[31] Jasty M, Maloney WJ, Bragdon CR, et al. The initiation of failure in cemented femoral components of hip arthroplasties. J Bone Joint Surg Br 1991; 73-B: 551-8.

[32] Carlsson AS, Nilsson JA, Blomgren G, Josefsson G, Lindberg LT. Low $v s$ high viscosity cement in hip arthroplasty. No radiographic difference in 226 arthrosis cases followed for 5 years. Acta Orthop Scand 1993; 64: 257-62.

[33] Mjoberg B, Ranzen H, Selvik G. Early detection of prosthetic-hip loosening. Comparison of low and high viscosity bone cement. Acta Orthop Scand 1990; 61: 273-74.

[34] Nelissen RG, Garling EH, Valstar ER. Influence of cement viscosity and cement mantle thickness on migration of the Exeter total hip prosthesis. J Arthroplasty 2005; 20: 521-8

[35] Havelin L1, Espehaug B, Vollset SE, Engesaeter LB. The effect of the type of cement on early revision. J Bone Joint Surg 1995; 77-A $1543-50$

[36] Ling RSM, Lee AJC. Porosity reduction in acrylic cement is clinically irrelevant. Clin Orthop 1998; 355: 249-53.

[37] Hernigou P, Le Mouël S. Do voids in a femoral cement mantle affect the outcome? J Arthroplasty 1999; 14: 1005-11.

[38] Malcom AJ. Pathology of low frictionarthroplasties in autopsy specimens. In older MWJ Ed. Implant bone interface. London, Springer-Verlag 1990; pp. 77-82.

[39] Brooks DB, Burstein AH, Frankel VH. The biomechanics of torsional fractures. The stress concentration effect of a drill hole. Bone Joint Surg 1970; 52-A: 507-14.

[40] Roberts EB, Kadakia NR, Noble PC, Carlyle TA, Tullos HS. Do cement pores contribute to mantle fractures in cemented total hip arthroplasties. Orthop Trans 1997; 21: 106.

[41] Mann KA, Gupta S, Race A, et al. Cement microcracks in thinmantle regions after in vitro fatigue load. J Arthroplasty 2004; 19 605-12.

[42] Qi G, Li J, Mouchon WP, Lewis G. Defect-induced fatigue microcrack formation in cement mantle. Biomed Mater Res A 2005; 75: 414-24.

[43] Herberts P, Malchau H. How outcome studies have changed total hip arthroplasty practices in Sweden. Clin Orthop 1997; 344: 4460 .

[44] Schulte KR, Collagan JJ, Kelley SS, Johnston RC. The outcome of Charnley total hip arthroplasty with cement after a minimum twenty year follow up. J Bone Joint Surg 1993; 75-A: 961-75.

[45] Rasquinha VJ, Dua V, Rodriguez JA, Ranawat CS. Fifteen-Year survivorship of a collarless, cemented normalized femoral stem in 
primary hybrid total hip arthroplasty with a modified thirdgeneration cement technique. J Arthroplasty 2003; 18: 86-94.

[46] Wroblewski BM, Siney PD, Fleming PA. Triple taper polished cemented stem in total hip arthroplasty: rationale for the design, surgical technique, and 7 years of clinical experience. J Arthroplasty 2001; 16(Suppl 1): 37-41.

[47] Chiu KH, Shen WY, Cheung KW, Tsui HF. Primary Exeter total hip arthroplasty in patients with small femurs: a minimal of 10 years follow-up. J Arthroplasty 2005; 20: 275-81.
[48] Glyn-Jones S, Gill HS, Beard DJ, McLardy-Smith P, Murray DW. Infleunce of stem geometry on the stability of polished tapered cemented femoral stems. J Bone Joint Surg Br 2005; 87-B: 921-7.

[49] Berli BJ, Schäfer D, Morscher EW. Ten-Year survival of the MS30 matt-surfaced cemented stem. J Bone Joint Surg Br 2005; 87-B: 928-33.

(C) Hernigou et al.; Licensee Bentham Open.

This is an open access article licensed under the terms of the Creative Commons Attribution Non-Commercial License (http: //creativecommons.org/licenses/by$\mathrm{nc} / 3.0 /$ ) which permits unrestricted, non-commercial use, distribution and reproduction in any medium, provided the work is properly cited. 